\title{
Colonization and infection in the newborn infant: Does chlorhexidine play a role in infection prevention?
}

\author{
Lizeth Ortegón, M.D. ${ }^{a}$, Marcela Puentes-Herrera, M.D. ${ }^{b}$, Ivohne F. Corrales, M.D. ${ }^{c}$ \\ and Jorge A. Cortés, M.D. ${ }^{b}$
}

\begin{abstract}
Healthcare-associated infections are a major problem in newborn infants, considering their high morbidity, mortality, and long-term sequelae. In preterm infants, it has been shown that skin and gastrointestinal tract colonization undergoes variations compared to healthy term infants, and that preterm infants are more exposed to nosocomial microorganisms given their higher probability of being admitted to the neonatal intensive care unit where they are cared for.

This document reviews normal colonization, the changes observed during hospitalization, prematurity, and the potential roleof chlorhexidine in the prevention of resistant microorganism transmission, as well as its side effects in newborn infantsadmitted to theneonatal intensivecareunit. Keywords: preterm infant, chlorhexidine, nosocomial infection, sepsis, primary prevention.
\end{abstract}

http://dx.doi.org/10.5546/aap.2017.eng.65

a. Universidad del Rosario, Neonatology Program, Bogotá, Colombia.

b. Department of Internal Medicine and Group of Research in Infectious Diseases, School of Medicine, Universidad Nacional de Colombia, Bogotá, Colombia.

c. Unit of Neonatology, Fundación Cardioinfantil, Bogotá, Colombia.

E-mail address:

Jorge A. Cortés, M.D.: jacortesl@unal.edu.co

Funding:

None.

Conflict of interest: None.

Received: 2-19-2016 Accepted: 8-12-2016
Healthcare-associated infections (HAIs) in newborn infants have a great impact in terms of morbidity, mortality, costs and long-term sequelae. Several practices have demonstrated their effectiveness to reduce HAIs in neonatal intensive care units (NICUs), such as hand washing, central venous catheter care protocols, etc. ${ }^{1}$ However, HAIs are still a major problem among hospitalized newborn infants, especially preterm infants.

Finding preventive measures to reduce the incidence of nosocomial sepsis at the NICUs has become a clinical research priority and a big challenge for health care providers working there. ${ }^{2}$ Some strategies have not been successful, whereas others such as nutrition, prophylaxis, and probiotics have shown good results in specific settings (e.g., enterocolitis). The use of heparin and antibiotic-impregnated catheters for the prevention of device-associated infections has been successful in some hospitals, but it has not been routinely standardized. $^{3}$

Considering that, since the moment of birth, newborn infants start acquiring a normal flora that is altered in the hospital setting, the colonization range of newborn infants admitted to the NICU may include potentially pathogenic flora, which may result in serious infections. Therefore, the potential prevention of hospital flora colonization through skin disinfection is of great interest.

The following review will describe the normal colonization process, and its alterations, in the newborn infant, as well as the potential role of chlorhexidine for infection prevention.

\section{Colonization of the gastrointestinal tract}

Studying gastrointestinal colonization in newborn infants is critical to understand the role of microbiome in the pathogenesis of neonatal sepsis. ${ }^{4}$ The gut microbiome should be recognized as a community of commensal, symbiotic and pathogenic microorganisms, as a super organ in itself, resulting from the interaction of microbial genes and a host that plays an essential role in immune system maturation and infections. ${ }^{5}$

The intestinal flora contains more than 400 microbial species and is acquired around 24-36 months of life. The acquisition of microbial patterns is the result of large intra- and inter-personal variations secondary to multiple external factors that affect neonates and infants. ${ }^{5-7}$ The environment surrounding the fetus 
is sterile, so is their gut, which is immersed in amniotic fluid. In healthy newborn infants, bacterial colonization starts during birth and through exposure to the maternal vaginal, fecal and skin flora, as well as environmental flora; this contact enables the development of the initial microbiome. ${ }^{5}$

The delivery mode shapes the initial gut microbiota. ${ }^{9}$ Such flora is different among infants born by C-section, even at 6 months old, and provides a different immunological response than that of vaginally delivered infants. In the latter, the most commonly isolated microorganisms are Lactobacillus spp. and Prevotella spp.; whereas in neonates born by C-section, they include Staphylococcus spp., Propionibacterium spp., and Corynebacterium spp. ${ }^{5,10}$ In other studies, it has been observed that among neonates born by abdominal surgery, the most commonly isolated microorganisms included Clostridium spp. and different Bifidobacterium species, and no differences were established in the frequency of their isolation compared to those delivered vaginally. ${ }^{9}$

The main source of neonatal gut colonization is maternal flora; however, some studies have shown that a third of newborn infants have a very low maternal flora colonization rate. ${ }^{9}$

A study compared fecal samples of mothers, nurses and healthy newborn infants and showed, after a 6-month follow-up, that there was no statistically significant difference in the prevalence of maternal strains over those of nurses in terms of newborn infant colonization; this suggests the importance of early contacts between neonates and their microbiological environment. ${ }^{9}$ Diet is also an influential factor: breastfed term infants have a higher Bifidobacteriaceae and Bacteroides spp. colonization compared to formula-fed infants, who have a higher Enterobacteriaceae colonization since their first day of life $\mathrm{e}^{5,10,11}$ (Table 1).

Preterm infants have a different colonization process due to organ immaturity, frequent antibiotic use, and prolonged hospital stays. These newborn infants show lower species diversity and a higher rate of potentially pathogenic microorganisms. In addition to Enterococcus, Bifidobacterium, Lactobacillus, and Bacteroides species, preterm infants are more commonly colonized by Klebsiella pneumoniae, C. difficile and C. perfringens. ${ }^{12}$ In preterm infants who require prolonged parenteral nutrition, the most commonly isolated microorganism was coagulase-negative staphylococcus (CoNS), in addition to Escherichia coli, Klebsiella spp., Enterobacter spp., and Serratia marcescens. ${ }^{13}$ It may be argued that, in addition to using parenteral nutrition, lack of breastfeeding is a risk factor for pathogenic colonization.

\section{Colonization as a predictor of neonatal sepsis}

Unlike early-onset neonatal sepsis, for which the amniotic cavity and the birth canal have been identified as the sources of the main etiologic agents, the origin of late-onset sepsis is more complex and extended. In recent years, the hypothesis of bacterial flora dissemination from the gastrointestinal tract as a source of bacteremia and sepsis, as a result of abnormal colonization in newborn infants at risk, has gained impetus ${ }^{9-14}$ (Table 2).

Most newborn infants hospitalized with suspected sepsis are managed with both broad-spectrum antibiotics and a delay in or contraindication for enteral nutrition, thus altering the normal colonization process and mucous membrane integrity. ${ }^{15,16}$ It has been proposed that, in these cases, the non pathogenic flora that usually colonizes newborn infants is replaced with potentially pathogenic and multidrug resistant microorganisms, which, in addition to an increased patency in microbial components, may be related to the development of necrotizing enterocolitis, sepsis, multiple organ failure, and refractory shock. ${ }^{17}$

TABLE 1. Colonization of the gastrointestinal tract in newborn infants based on diet ${ }^{5,8,14}$

\begin{tabular}{lll}
\hline & Exclusive breastfeeding & Milk formula \\
\hline First day & $\begin{array}{l}\text { Streptococcus spp., Staphylococcus spp. } \\
\text { and Enterococcus spp. }\end{array}$ & Enterobacteriaceae. \\
First week & $\begin{array}{l}\text { Bifidobacteriaceae. } \\
\text { (1000:1 ratio compared to Enterobacteriaceae). }\end{array}$ & $\begin{array}{l}\text { Bifidobacteriaceae. } \\
\text { First month }\end{array}$ \\
& Clostridium spp. (6\%-20\%). & Bifidobacteriaceae (reduced from $71 \%$ to $64 \%)$. \\
& Bacteroides spp. & Enterococci (increased from $57 \%$ to $86 \%)$. \\
& & Clostridium spp. (50\%). \\
\hline
\end{tabular}


One study compared three groups of preterm newborn infants: the first group (pre-colonized) included patients diagnosed with sepsis; the second group (overlapping) included patients not diagnosed with sepsis but who shared time and space with the first group; and the third group included patients randomized over time. It was observed that the same microorganisms were isolated in some of the patients in the overlapping group and the pre-colonized group, which demonstrated inter-patient transmission within the neonatal care unit. The most commonly isolated agent in relation to sepsis was group B Streptococcus. ${ }^{14}$

Another study compared a group of preterm infants diagnosed with late-onset sepsis and a control group. CoNS was isolated in $70 \%$ of newborn infants who had sepsis, with no differences versus the control group. A higher rate of Propionibacterium was observed in the control group. This suggests that the absence of certain non pathogenic microorganisms -rather than the presence of pathogenic microorganisms in itself- may be one of the risk factors for the development of neonatal sepsis. ${ }^{18}$

A study carried out in low birth weight newborn infants at a NICU showed that the risk factor for multi-drug resistant Enterobacteriaceae was prior maternal colonization. ${ }^{19}$

\section{Skin colonization}

The characteristics of newborn infants' skin increase their risk for infections and pose a risk for potential substance absorption, making the skin more susceptible to local damage and water loss. ${ }^{20}$ Although the skin of healthy term newborn infants is very similar to that of adults in terms of skin and horny layer thickness, the dermis and epidermis are not fully developed and therefore require a neonatal adaptation process, just like the lipid barrier. ${ }^{20}$ Such maturation process is facilitated by the colonization of symbiotic, non pathogenic bacteria in healthy term infants. ${ }^{21}$

The prevalent bacterial population in newborn infants are aerobic species, including Staphylococcus and Corynebacterium, and anaerobic organisms such as Propionibacterium. In preterm infants, CoNS account for $80 \%$ of the total flora, with a highly heterogeneous distribution, predominantly in the navel, skin folds, buttocks and soles. The transient flora is variable and depends on the child's environment. Enterococci, Enterobacteriaceae, Acinetobacter spp., Pseudomonas aeruginosa may be isolated in the skin and become opportunistic pathogenic microorganisms and result in healthcare associated infections. ${ }^{10,22}$

The most commonly implicated microorganisms in the etiology of nosocomial sepsis are those that colonize the skin, the gastrointestinal tract, the mucous membranes, and the different monitoring and support devices. Gram-positive microorganisms are responsible for $83 \%$ of HAIs; among these, the most common include CoNS and Staphylococcus aureus, followed by K. pneumoniae, E. coli, P. aeruginosa, Enterobacter spp., Acinetobacter spp., and the different Candida species. ${ }^{23}$

\section{The role of chlorhexidine}

Skin disinfection is one of the best methods to prevent infections related to invasive procedures. Umbilical cord care is equally relevant, an area that has been described as the most sensitive site for bacterial colonization, mainly Staphylococcus aureus. ${ }^{24}$

Chlorhexidine is a powerful, broad-spectrum antimicrobial antiseptic. It is bactericidal against Gram-positive microorganisms, inhibits spore

TABLE 2. Colonization of the gastrointestinal tract in newborn infants with and without sepsis ${ }^{9-13,15-19}$

\begin{tabular}{ll}
\hline Normal flora & Microorganisms responsible for late-onset sepsis \\
\hline Lactobacillus spp. & Coagulase-negative Staphylococcus \\
Prevotella spp. & Staphylococcus aureus \\
Enterobacteriaceae & Escherichia coli \\
Streptococcus spp. & Klebsiella spp. \\
Staphylococcus spp. & Pseudomonas spp. \\
Enterococcus spp. & Group B Streptococcus \\
Clostridium spp. & Candida spp. \\
Bacteroides spp. & Serratia spp. \\
& Acinetobacter spp. \\
& Enterobacter spp. \\
\hline
\end{tabular}


growth, and is bacteriostatic against mycobacteria. It also inactivates lipid-enveloped viruses, such as herpes and human immunodeficiency virus (HIV). Chlorhexidine activity is practically unaffected by the presence of blood or organic material, and it may be used on the skin even if skin integrity is damaged, without affecting the wound healing process. It was observed that the effectiveness of $0.5 \%$ chlorhexidine in $70 \%$ isopropyl alcohol was similar to that of povidone iodine without the side effects related to the thyroid function and burns to the skin. ${ }^{25}$

Chlorhexidine may be supplied in different dosage forms, and the most popular ones include $2 \%$ chlorhexidine in $70 \%$ isopropyl alcohol, $4 \%$ water-based chlorhexidine, and $0.5 \%$ chlorhexidine in $70 \%$ isopropyl alcohol. In newborn infants, $0.5 \%-1 \%$ chlorhexidine gluconate is the antiseptic of choice for skin disinfection. ${ }^{25}$ Several studies assessed the effect of birth canal cleansing in association with whole-body bathing in newborn infants and showed reduced mortality, unlike those who only underwent whole-body bathing with no intervention at the birth canal. ${ }^{26}$

A meta-analysis of clinical trials that assessed umbilical cord care protocols showed a $23 \%$ reduction in neonatal mortality and a $27 \%$ $56 \%$ reduction in omphalitis in the group using chlorhexidine. ${ }^{27}$ Another meta-analysis demonstrated a reduction in mortality and in the incidence of omphalitis among patients who received umbilical cord care with chlorhexidine versus routine community care. But such reduction was not sustained versus routine hospital care or in patients whose skin was cleansed with chlorhexidine compared to control patients. ${ }^{28}$ This effect has been observed in developing countries with a high prevalence of neonatal sepsis and sepsis-associated mortality, but not in those with a low sepsis prevalence. ${ }^{29}$

\section{Chlorhexidine adverse reactions}

In 2009, a survey was administered to the directors of neonatology training programs in the United States. As per results, chlorhexidine was used in $61 \%$ of neonatology units, although its use was not approved by the Food and Drug Administration (FDA). Among these, 51\% reported skin reactions, including erythema $(32 \%)$ and erosions (7\%). No systemic toxicity or neurological toxicity were reported..$^{30}$

In fact, a literature review suggests that chlorhexidine in concentrations above $2 \%$ causes acidic symptoms. In analytical clinical trials, chlorhexidine-impregnated dressings used to cover percutaneous catheter insertion sites have been reported to cause contact dermatitis in almost $5.7 \%$ of patients under 26 weeks of gestational age. ${ }^{31}$

The rate of exanthema depends on different factors, such as gestational age, skin hypersensitivity, and the different concentrations of chlorhexidine used; however, in general, it may occur in $5 \%$ of all newborn infants and in $15 \%$ of preterm infants with a weight of less than 1000 grams and born with less than 28 weeks of gestation. ${ }^{25}$

Studies that used whole-body chlorhexidine bathing as the main intervention did not evidence a significant increase in the risk of exanthema in newborn infants using $0.6 \%$ chlorhexidine in sterile water. ${ }^{32}$

The evidence regarding the side effects of buildup in the blood of infants cleansed with chlorhexidine-impregnated cloths on a daily basis is not clear. It is not possible to compare studies available in the literature because no trial has been conducted using standardized sampling. The higher reported levels have been attributed to contamination that may have occurred during heel blood sampling versus puncture blood sampling. No correlation has been established between blood levels and subjects' age, time of use, or the number of prior baths. ${ }^{31,33}$ There are case series that reported chlorhexidine accidental intake, and side effects resolved once exposure was interrupted..$^{34,35}$

\section{CONCLUSIONS}

Considering that skin colonization with potentially pathogenic microbial flora typical of the hospital setting is a risk factor for healthcareassociated infections, and topical chlorhexidine has demonstrated to reduce skin colonization rates in the skin of patients with potentially pathogenic microorganisms with minimal side effects, chlorhexidine bathing may be an effective, easily applicable, and low cost strategy for newborn infants hospitalized at the NICU who have a high risk of nosocomial infections. We recommend using $0.5 \%-1 \%$ chlorhexidine, which has proven to be safe for the neonatal population. Although 2\% chlorhexidine is considered category IA by the Centers for Disease Control and Prevention (CDC), it requires to be validated in the neonatal population. Further studies are required to standardize this intervention, 
especially among preterm and low birth weight newborn infants.

At present, the main use of alcohol- or waterbased $2 \%$ chlorhexidine skin preparations is skin disinfection prior to peripheral vascular device and central venous line insertion. These preparations are also used to disinfect the skin prior to invasive or surgical procedures. Another common use for alcohol- or water-based $2 \%$ chlorhexidine is umbilical cord care both in the hospital setting and at a community level. At the intensive care unit, chlorhexidine bathing is preferably done using chlorhexidine dilutions at $1 \%$ or lower; it is used in term and preterm stable newborn infants during their weight gain period, in general, if they are at least two weeks old and were born after at least 28 weeks of gestation with a birth weight of more than 1500 grams.

\section{REFERENCES}

1. Guía de prevención, vigilancia epidemiológica y control de las infecciones asociadas al cuidado de la salud en las unidades de recién nacidos. Bogotá: Secretaría Distrital de Salud, 2011. [Accessed on: August 16 ${ }^{\text {th }}, 2016$ ]. Available at: http://www.saludcapital.gov.co/sitios/ VigilanciaSaludPublica/Todo $\% 20 \mathrm{IIH} / \mathrm{Gu} \% \mathrm{C} 3 \% \mathrm{ADa} \% 20$ Recien $\% 20$ Nacido\%20pdf.pdf.

2. Stronati M, Bollani L, Maragliano R, Ruffinazzi G, et al. Neonatal sepsis: new preventive strategies. Minerva Pediatr 2013;65(1):103-10.

3. Shah $\mathrm{P}$, Shah V. Continuous heparin infusion to prevent thrombosis and catheter occlusion in neonates with peripherally placed percutaneous central venous catheters. Cochrane Database Syst Rev 2005(3):CD002772.

4. Eggesbo M, Moen B,PeddadaS, Baird D, etal. Development of gut microbiota in infants not exposed to medical interventions. APMIS 2011;119(1):17-35.

5. Madan JC, Farzan SF, Hibberd PL, Karagas MR. Normal neonatal microbiome variation in relation to environmental factors, infection and allergy. Curr Opin Pediatr 2012;24(6):753-9.

6. Favier CF, Vaughan EE, De Vos WM, Akkermans AD. Molecular monitoring of succession of bacterial communities in human neonates. Appl Environ Microbiol 2002;68(1):219-26.

7. Yatsunenko T, Rey FE, Manary MJ, Trehan I, et al. Human gut microbiome viewed across age and geography. Nature 2012;486(7402):222-7.

8. FanaroS,Chierici R, Guerrini P, Vigi V. Intestinal microflora in early infancy: composition and development. Acta Paediatr Suppl 2003;91(441):48-55.

9. Gronlund MM, Lehtonen OP, Eerola E, Kero P. Fecal microflora in healthy infants born by different methods of delivery: permanent changes in intestinal flora after cesarean delivery. JPediatr Gastroenterol Nutr 1999;28(1):1925.

10. Dominguez-BelloMG, CostelloEK,Contreras M,Magris M, et al. Delivery mode shapes the acquisition and structure of the initial microbiota across multiple body habitats in newborns. Proc Natl Acad Sci U S A 2010;107(26):11971-5.

11. Guaraldi F, SalvatoriG. Effect of breast and formula feeding on gut microbiota shaping in newborns. Front Cell Infect Microbiol 2012;2:94.
12. Arboleya S, Binetti A, Salazar N, Fernández N, et al. Establishment and development of intestinal microbiota in preterm neonates. FEMS Microbiol Ecol 2012;79(3):76372.

13. Fernandes PC, Dolinger EJ, Abdallah VO, Resende DS, et al. Late onset sepsis and intestinal bacterial colonization in very low birth weight infants receiving long-term parenteral nutrition. Rev Soc Bras Med Trop 2011;44(4):447-50.

14. Carl MA, Ndao IM, Springman AC, Manning SD, et al. Sepsis from the gut: the enteric habitat of bacteria that cause late-onset neonatal bloodstream infections. Clin Infect Dis 2014;58(9):1211-8.

15. Sharma R, Young C, Neu J. Molecular modulation of intestinal epithelial barrier: contribution of microbiota. J Biomed Biotechnol 2010;2010:305879.

16. Mshvildadze M, Neu J, Mai V. Intestinal microbiota development in the premature neonate: establishment of a lasting commensal relationship? Nutr Rev 2008;66(11):65863.

17. Claud EC, Walker WA. Hypothesis: inappropriate colonization of the premature intestine can cause neonatal necrotizing enterocolitis. FASEB J 2001;15(8):1398-403.

18. Mai V, Torrazza RM,Ukhanova M, WangX, etal. Distortions in development of intestinal microbiota associated with late onset sepsis in preterm infants. PLoS One 2013;8(1):e52876.

19. Denkel LA, Schwab F, Kola A, Leistner R, et al. The mother as most important risk factor for colonization of very low birth weight (VLBW) infants with extended-spectrum beta-lactamase-producing Enterobacteriaceae (ESBL-E). J Antimicrob Chemother 2014;69(8):2230-7.

20. Hoeger PH, Enzmann CC. Skin physiology of the neonate and young infant: a prospective study of functional skin parameters during early infancy. Pediatr Dermatol 2002;19(3):256-62.

21. Visscher MO, Chatterjee R, Munson KA, Pickens WL, et al. Changes in diapered and nondiapered infant skin over the first month of life. Pediatr Dermatol 2000;17(1):45-51.

22. CaponeKA, DowdSE, Stamatas GN, NikolovskiJ. Diversity of the human skin microbiome early in life.J Invest Dermatol 2011;131(10):2026-32.

23. Vergnano S, Sharland M, Kazembe P, Mwansambo C, et al. Neonatal sepsis: an international perspective. Arch Dis Child Fetal Neonatal Ed 2005;90(3):F220-4.

24. Oishi T, Iwata S, Nonoyama M, Tsuji A, et al. Double-blind comparative study on the care of the neonatal umbilicalcord using $80 \%$ ethanol with or without chlorhexidine. J Hosp Infect 2004;58(1):34-7.

25. Montes MT, AresS,Sola A. Recomendaciones dela Sociedad Iberoamericana de Neonatología (SIBEN). Consenso Utilización de soluciones antisépticas en recién nacidos. 2008. [Accessed on: August 16 ${ }^{\text {th }}$,2016]. Available at: http:/ / www.codeinep.org/restricted/antisepticos_en_nn.pdf.

26. Sankar MJ, Paul VK. Efficacy and safety of whole body skin cleansing with chlorhexidine in neonates--a systemic review. Pediatr Infect Dis J 2013;32(6):e227-34.

27. Imdad A, Bautista RM, Senen KA, Uy ME, et al. Umbilical cord antiseptics for preventing sepsis and death among newborns. Cochrane Database Syst Rev 2013;(5):CD008635.

28. Sinha A, SazawalS, Pradhan A, RamjiS, etal. Chlorhexidine skin or cord care for prevention of mortality and infections in neonates. Cochrane Database Syst Rev 2015;(3):CD007835.

29. Da Cunha ML, Procianoy RS. Effect of bathing on skin flora of preterm newborns. J Perinatol 2005;25(6):375-9.

30. Watkins AM, Keogh EJ. Alcohol burns in the neonate. J Paediatr Child Health 1992;28(4):306-8.

31. Visscher M, deCastro MV, Combs L, Perkins L, et al. Effect of chlorhexidine gluconate on the skin integrity at PICC line sites. J Perinatol 2009;29(12):802-7. 
70 / Arch Argent Pediatr 2017;115(1):65-70 / Special article

32. Saleem S, Rouse DJ, McClure EM, Zaidi A, et al. Chlorhexidine vaginal and infant wipes to reduce perinatal mortality and morbidity: a randomized controlled trial. Obstet Gynecol 2010;115(6):1225-32.

33. Lee A, Harlan R, Breaud AR, Speck K, et al. Blood concentrations of chlorhexidine in hospitalized children undergoing daily chlorhexidine bathing. Infect Control Hosp Epidemiol 2011;32(4):395-7.
34. Mucklow ES. Accidental feeding of a dilute antiseptic solution (chlorhexidine $0.05 \%$ with cetrimide $1 \%$ ) to five babies. Hum Toxicol 1988;7(6):567-9.

35. Balit CR, Lynch AM, Gilmore SP, Murray L, et al. Lignocaine and chlorhexidine toxicity in children resulting from mouth paint ingestion: a bottling problem. J Paediatr Child Health 2006;42(6):350-3. 